\title{
ADAPTIVE CRITIC FOR PROBABILISTIC LOGIC NETS
}

\author{
R.S.Neville and T.J.Stonham \\ Department of Electrical Engineering, Brunel University, Uxbridge UB8 3PH, U.K.
}

\begin{abstract}
In this paper we study the use of an Adaptive Critic to augment Probabilistic Logic nets' performance. The paper proposes a new Quantised Adaptive Critic Element for use with Probabilistic Logic Nodes(PLN). The Probabilistic units are logical nodes which respond to their input pattems in addressable locations; the locations then define the probability of the output being a logical " 1 ". The technique uses an Unsupervised Adaptive Critic Element to predict a Reward signal for the net. The prediction is based on the standard scalar Reward, and the past and present predicted values of the reinforcement signal. The Quantised Adaptive Critic methodology may be utilised for problems which involve a large number of input variables(i.e. control). Anew structure is proposed to deal with this situation and enable the Adaptive Critic to be Hardware realisable.
\end{abstract}

\section{Quantised Adaptive Critic}

The Quantised Adaptive Critic Element (QACE) stores the variables that it utilises in the same manner as the PLN, Neville [1993]. The QACE has a site address $v$ which is addressed by an $i$ bit vector $\left\{x_{1}, x_{2}, \ldots x_{i}\right\}$, this addresses three sites in parallel $P_{v_{(t)}}, P_{v_{(1-1)}} \in\left[+P_{n}, 0\right]$ and $\bar{x}_{v_{(t)}} \in\left[+\bar{x}_{n}, 0\right]$, that are $q$ bit numbers. The QACE uses the standard external reward signal $r=1-e_{o}$ where $r \in[+1,0]$ is a binary scalar value. The reinforcement is then scaled $r^{\prime}=(2 * r)-1$ to obtain the reinforcement used by Barto et al.[1983], where $r^{\prime} \in[0,-1]$. The scaled reward signal is then used to derive an improved or internal reinforcement signal, given by: $\hat{r}_{(t)}=r_{(t)}{ }^{\prime}+\gamma P_{v_{(t)}}-P_{v_{(t-1)}}$

where $P_{v_{(t)}}$ is the present prediction and $P_{v_{(t-1)}}$ is the past prediction. One should note that this is not the same as Barto's work, where he uses the prediction values $P_{(t)}$ and $P_{(t-1)}$. We use the present and previous prediction values for the given site address $v$. The Prediction value is updated by: $\Delta P_{v_{(t+1)}}=\beta \hat{r} \bar{x}_{v_{(t)}}$

where $\beta$ is a positive constant determining the rate of change of $P_{v}$. All the input eligibility traces are updated by: $\bar{x}_{v_{(t+1)}}=\lambda \bar{x}_{v_{(l)}}+(1-\lambda) x_{v}$

where $\lambda, 0<\lambda<1$ determines the eligibility trace decay rate. The binary scalar value $x_{v}$ is a trigger for the eligibility trace, when the site $v$ is addressed $x_{v}=1$ and all other non-addressed traces are updated with $x(\bar{v})=0$. The internal reinforcement $\hat{r}$ is then re-scaled $r^{*}=(\hat{r}+1.0) / 2.0$ which denote a quantised real valued reinforcement $0.0<r^{*}<1.0$. The addressed sites are then adapted using: $\Delta S_{\mu}=r^{*} \alpha\left[Y-\sigma\left(S_{\mu}\right)\right]+\left(1-r^{*}\right) \alpha \lambda[1-Y-\sigma(S \mu)]$

\section{Concluding Remarks}

In this work we have put forward a Quantised Adaptive Critic methodology for augmenting Probabilistic Logic Nets. The unsupervised Critic provides advisory information in the form of a quantised internal reinforcement, which promotes learning efficiency. The standard Adaptive Critic methodology has a single Critic as advisor, while we find it advantageous to have one Critic per layer to augment the training of the supervised net. An alternative approach to obtain the internal reinforcement was the use of the multicube structure. The use of several cubes bypasses the problem of exponentially growing resources with increased fan-in. The quantisation of the prediction and eligibility values enables the Quantised Adaptive Critic structure to be hardware realisable. One should note that in this study we have used the Critic to predict the present internal reinforcement $\hat{r}_{(t)}$, the Critic is also able to predict the future reinforcement at $\hat{r}_{(t+1)}$, which may be more useful in temporal or control situations. In order to predict $\hat{r}_{(t+1)}$ one would utilise the future prediction $P v_{(t+1)}$ and the present prediction $P v_{(t)}$

\section{References}

Barto, A.G., Sutton, R.S., Anderson, C.W. (1983). Neuron like Adaptive Elements that can solve difficult leaming control problems. IEEE Transactions on, Man, and Cybernetics, Volume SMC-13, Number 5, Septemher/Octoher, pp834-846.

Neville. R.S. (199.3). Adaptive Critic for Probahilistic Logic Nets. World Congress on Neural Networks (WCNN'93 Portland), July 11-15, Portland, Oregon, USA. 